\title{
LA RELEVANCIA DE LAS FINANZAS EN UN MODELO DE NEGOCIO SOSTENIBLE EN POBLACIONES VULNERABLES
}

\author{
THE RELEVANCE OF FINANCE WITHIN A SUSTAINABLE BUSINESS MODEL IN \\ VULNERABLE POPULATIONS
}

\section{RESUMEN}

Objetivo: Analizar la relevancia de las finanzas en un modelo de negocio sostenible en poblaciones vulnerables. Método: Se realizó una investigación cualitativa, basada en la revisión de literatura incluyendo los conceptos de sostenibilidad, finanzas sostenibles, cadenas de valor, cambio climático y economía circular. Se incluyó principalmente la revisión de bases bibliográficas como Scopus, Clarivate, Academia y Google Académico, y se excluyó la revisión de literatura de las finanzas clásicas. Resultados: Se identificó que los aspectos financieros son incluidos en la gestión de los negocios sostenibles; sin embargo, estos se realizan de forma convencional dificultando que alcancen los fondos necesarios. Se propone un modelo que es abordado desde una perspectiva de cadena de valor que considera la gestión de los riesgos ambientales, sociales y económicos, buscando que quienes más riesgos enfrentan reciban mayor rendimiento. Conclusión: Para lograr que se implante un modelo conceptual, en el que las finanzas sostenibles tengan una clara participación, se considera relevante asegurar un mayor empoderamiento a las poblaciones a quienes se busca favorecer a través de una mejor formación financiera; e igualmente, es clave que las fuentes de financiamiento profundicen la evaluación de los riesgos y rendimientos, y los resultados sean medibles en los estados financieros de los negocios.

Palabras clave: Sostenibilidad; economía circular; cadenas de valor; finanzas.
Walther Hernán Reátegui Vela Universidad ESAN Lima, Perú

ORCID: https://orcid.org/0000-0003-0005-3113 Correo electrónico: wreategui@esan.edu.pe

Ana Inés Reátegui Vela Universidad ESAN Lima, Perú

ORCID: https://orcid.org/0000-0003-3395-652X Autor para correspondencia: areategui@esan.edu.pe

\begin{abstract}
Objective: Analyze the relevance of the finance within a sustainable business model in vulnerable population. Method: A qualitative research was carried out, based on the review of the applicable literature including the concepts of sustainability, sustainable finance, value chains, climate change and circular economy. Also, it included the review of bibliographic databases such as Scopus, Clarivate, Academia, and Google Scholar. The literature review of classical finance was excluded. Results: It has been identified that financial aspects are included in the management of sustainable business; however, these are carried out in a conventional way, making it difficult for them to reach the necessary funds. A model is proposed, which is approached from a value chain perspective and considers the management of environmental, social and economic risks, seeking that those who face the most risk receive the highest return. Conclusion: In order to achieve that a conceptual model is implanted, in which sustainable finance has a clear participation, it is relevant to ensure greater empowerment to the populations that are being favored through better financial training; likewise, it is essential that the financing sources deepen their evaluation concerning risks and returns, and that the results are measurable in the financial statements of each business.
\end{abstract}

Keywords: Sustainability; circular economy; value chains; finance.

( - Los autores. Este artículo es publicado por la revista Quipukamayoc de la Facultad de Ciencias Contables, Universidad Nacional Mayor de San Marcos. Este es un artículo de acceso abierto, distribuido bajo los términos de la licencia Creative Commons Atribución 4.0 Internacional (CC BY 4.0) [https://creativecommons.org/licenses by/4.0/deed.es] que permite el uso, distribución y reproducción en cualquier medio, siempre que la obra original sea debidamente citada de su fuente original. 


\section{INTRODUCCIÓN}

Si bien la sostenibilidad ha sido un concepto que durante los últimos años ha capturado la atención académica y profesional, se puede decir que la pandemia del COVID-19 ha mostrado la urgencia de implantar modelos de gerencia que la incorporen explícitamente. En este contexto, Geissdoerfer, Savaget, Bocken y Hultink (2017) proponen que la sostenibilidad es una transformación del estilo de vida que optimiza la seguridad, el bienestar y la salud.

Por su parte, Acero y Savaget (2014) indican que la sostenibilidad abre campo para múltiples expectativas sobre qué es sostenible, por cuánto tiempo y para qué personas sería beneficioso; por otro lado, aunque Bonini y Swartz (2014) comentan que la sostenibilidad puede ser vista como un lujo, esta visión es considerada infundada, pues la sostenibilidad puede ayudar a crear ganancias y oportunidades a todos los involucrados.

Sin embargo, si bien se ha puesto énfasis en evidenciar los cuidados que se debe tener en cuenta para generar una producción responsable, pareciera que el problema surge porque las finanzas no han sido abordadas dentro de un proceso integrador que apoye al desarrollo de los más vulnerables a pesar de su relevancia, sino que siguen siendo consideradas en forma ortodoxa, lo cual dificulta el acceso a los fondos. Al mantener una posición cerrada, las finanzas sostenibles no han tenido un campo de acción claro dentro de un modelo de negocios sostenible y, en especial, en favor de las poblaciones vulnerables.

Lo expresado en el párrafo previo coincide con Ziolo et al. (2019), quienes señalan que el desarrollo sostenible tiene como objetivo mitigar las externalidades negativas, y agregan que las finanzas convencionales no ofrecen espacio para el medioambiente y la sociedad, coincidiendo con Schoenmaker y Schramade (2018), y Bianchini y Gianfrate (2018). Reátegui (2021) profundiza esta idea, al señalar que las finanzas deben estar orientadas al desarrollo sostenible, a la sostenibilidad corporativa, a incentivar la actividad que promueve el crecimiento económico bajo en carbono - que privilegia la conservación de los recursos naturales y de la biodiversidad- y, en general, a aquellas actividades que enfrentan el cambio climático y la pobreza, en primera instancia.

Entonces, se puede decir que el desarrollo de las finanzas sostenibles es una herramienta que ayuda a mitigar el impacto de situaciones como la pandemia del COVID-19, que ha evidenciado la fuerte vinculación del cuidado ambiental y la salud, y que ha llevado al planeta a una de sus peores crisis. Prüss-Üstün, Corvalán y World Health Organization (2006) ya explicaban que el deterioro del medioambiente provocaba fuertes impactos en la salud, pero se puede agregar que hoy el planeta ha comprendido fehacientemente la relación entre medioambiente, salud y economía.

En este sentido, las finanzas sostenibles deben promover el direccionamiento de recursos financieros de inversionistas y fuentes de financiamiento directas hacia actividades económico-sostenibles de poblaciones vulnerables. Eso crearía capacidad de resiliencia frente a situaciones extremas e impulsaría el avance hacia el bienestar de estas poblaciones, superando sus restricciones estructurales, vía un modelo estructurado que concilia medioambiente, salud y economía, dejando menos espacio a eventos severos causados por el virus.

De igual forma, Kell y Rasche (2020) señalan que la pandemia ha mostrado que la salud humana, el bienestar económico y el medioambiente natural están profundamente conectados. Además, agregan que esta situación presenta una oportunidad única de aprender lecciones para el futuro.

De lo expresado se puede argumentar que la sostenibilidad es un tema clave, y que debe incorporarse en la gestión moderna de todo tipo de organizaciones, empezando con aquellas que nacen en las poblaciones vulnerables. Es decir, ocuparse de la sostenibilidad no debería ser visto como un costo, sino que su adecuada gestión puede generar réditos - para quienes estén directamente involucrados- y bienestar para la humanidad en general, siguiendo la línea de Bonini y Swartz (2014). Entonces, hablar de economía o finanzas en los temas de sostenibilidad es, a todas luces pertinente y a juicio de los autores, imprescindible, proponiendo para ello el modelo que se desarrolla en la presente investigación.

En este contexto, se desprende la siguiente pregunta: ¿Las finanzas son relevantes en un modelo de negocio sostenible en poblaciones vulnerables? De la brecha de conocimiento identificada, se establecen las siguientes categorías de análisis: poblaciones vulnerables ante el cambio climático, las finanzas al interior de las cadenas de valor para lograr una adecuada gestión de riesgos, y una contribución a que los negocios sean perdurables.

La limitación fundamental enfrentada para el desarrollo de este trabajo ha sido la escasa casuística de proyectos denominados "sostenibles" en los que se haga uso explícito de las finanzas sostenibles, tal como ha sido definida en esta investigación, y de un adecuado análisis de riesgo y rentabilidad.

De la literatura y de lo observado por los autores, se justifica la profundización y el mayor estudio de las acciones del ser humano realizadas en un contexto de cuidado del medioambiente, y en el que se generen oportunidades económicas para las poblaciones vulnerables, pero donde 
los elementos económico-financieros deben ser considerados por su relevancia absoluta. Como se expresó, si bien los elementos financieros son incluidos en los emprendimientos de las personas vulnerables, muchas veces se abordan desde una perspectiva clásica, sin la generación de un espacio para aplicar las finanzas sostenibles - aquellas que buscan asegurar fuentes de financiamiento a toda una cadena de valor sostenible (CVS) - en el que se reconozca que los riesgos que asumen los actores involucrados no son semejantes a una empresa tradicional. Se considera que, en los emprendimientos de las personas más frágiles, los mejores rendimientos no corresponden a quienes más riesgo enfrentan, lo que los envuelve en un círculo vicioso que les impide dejar la vulnerabilidad.

\section{MATERIAL Y MÉTODOS}

La investigación se abordó desde un enfoque cualitativo basado en la revisión de la literatura, así como en el conocimiento de los autores en proyectos en poblaciones vulnerables. Respecto a la literatura, se consultaron las bases bibliográficas de Scopus, Clarivate, Academia y Google Académico, sobre sostenibilidad, finanzas sostenibles, cadenas de valor, cambio climático, economía circular, entre otros.

Luego, la revisión de literatura fue confrontada con la experiencia de los autores de esta investigación en la implementación de proyectos realizados por poblaciones vulnerables. Sobre la base de lo mencionado, se propuso un esquema para la planificación de un negocio sostenible, como paso previo a la concepción del modelo conceptual de gestión.

\section{RESULTADOS}

Según lo expresado, se busca que las finanzas sostenibles se alineen con el objetivo de apoyar las inversiones de las personas y zonas con mayor vulnerabilidad frente al cambio climático y a una situación de adversidad; serán inversiones de impacto si promueven una inclusión económica eficaz a través de la mitigación y adaptación al cambio climático, de la conservación de la biodiversidad y de los recursos naturales, coincidiendo con las definiciones de sostenibilidad dadas por Ziolo et al. (2019), Schoenmaker y Schramade (2019), Bianchini y Gianfrate (2018), Acero y Savaget (2014), y Bonini y Swartz (2014), quienes vinculan al medioambiente con la sociedad en general y con los elementos económico-financieros. Para estos últimos elementos, surge la necesidad de que los mercados de capitales y las fuentes de financiamiento especializadas tengan un enfoque y capacidad para enfrentar el riesgo de las actividades económicas sostenibles, mejorando su mirada convencional respecto de los negocios, y reconociendo las diferencias sustanciales en la gestión de los riesgos.
No obstante, antes de mercados de capitales sostenibles, se requiere que los gestores de los emprendimientos sostenibles puedan mejorar sus competencias para que identifiquen y gestionen mejor los riesgos que enfrentan y, de esta manera, asignar una adecuada rentabilidad. Asimismo, deben tener un claro esquema de rendición de cuentas al interior de una economía colaborativa, ya que los operadores de los fondos dentro de una CVS no solo serán parte de la demanda, sino también creadores de valor que conservan el medioambiente - siguiendo a Nidumolu, Prahalad y Rangaswami (2009) - donde, según Reátegui (2021), cada actor obtendrá los beneficios económicos de acuerdo con el aporte que haga; asimismo, la fuente de financiamiento sostenible los reconocerá a la luz de un contrato único y mandatorio. Las personas vulnerables deben ser consideradas como parte de la demanda, pero también como potente factor de productos sostenibles.

La economía circular es otro concepto que ha logrado atraer el interés de quienes tienen la responsabilidad de generar políticas públicas, así como del sector privado. Ellen MacArthur Foundation (2013) presenta a la economía circular como una economía industrial que es restauradora o regenerativa por intención y diseño. Stahel (2019) señala que esta rama de la ciencia propone una nueva relación entre los bienes y los materiales, ahorrando recursos y minimizando desperdicios. Semieniuk, Campiglio, Mercure, Volz y Edwards (2021) señalan que la transición a una economía baja en carbono provocará cambios en la revalorización de los activos de la industria y en la estructura de los sistemas financieros.

Al unir los conceptos de sostenibilidad, cadena de valor, economía circular, cambio climático y vulnerabilidad surge la Figura 1, la cual muestra un esquema propuesto por los autores para la planificación de un negocio sostenible, incluyendo de manera específica a las finanzas, y considerando que es un paso previo a la concepción del modelo de gestión de negocios.

En la literatura, se hace referencia a las cadenas de valor, identificando que deben considerar la sostenibilidad política, sostenibilidad financiera, investigativa, ecológica, y humana (Barrientos, 2018). En el caso de la sostenibilidad financiera, se habla más bien de un financiamiento tradicional, sin considerar un cambio de condiciones hacia los más vulnerables en la cadena.

Se parte de reconocer, por ejemplo, la existencia de eventos de riesgo como heladas, sequías o lluvias intensas, que ponen en riesgo el ecosistema que rodea a productores (punto 1) y genera impactos negativos que acentúan la condición de pobreza de un segmento de la población (punto 2). En este escenario, la actividad económica sostenible, que a su vez parte de la gestión del riesgo climático, 


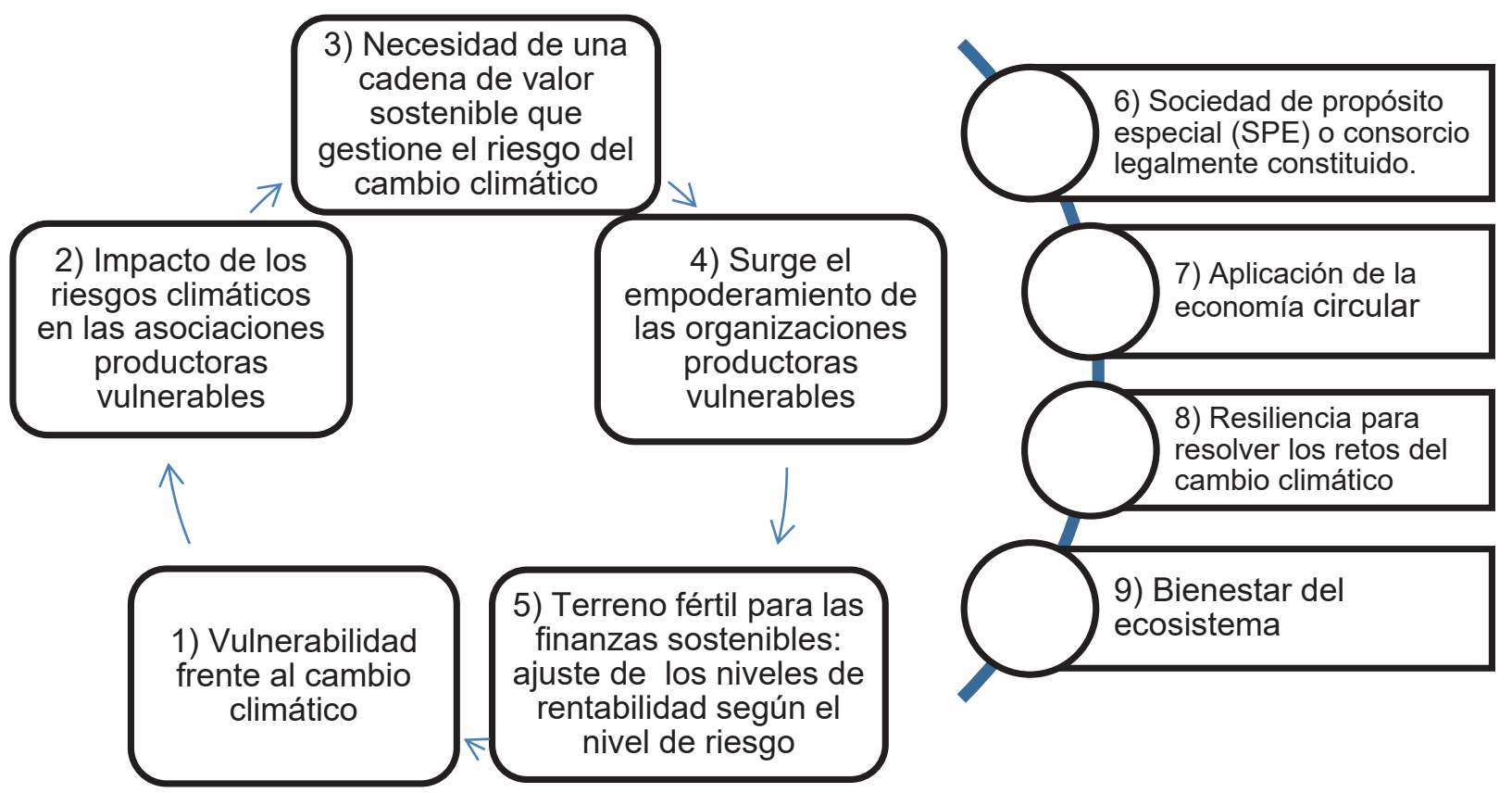

Figura 1. Planificación de un negocio sostenible

Fuente: Elaboración propia sobre la base de Reátegui (2021)

requiere ser reforzada por una cadena de valor (punto 3). En ese sentido, como primer resultado, surge el empoderamiento de la organización de productores vulnerables (punto 4).

Con esta perspectiva integradora, las finanzas sostenibles encuentran un terreno fértil, mejor ajustado a los riesgos de las actividades económicas sostenibles (punto 5). Así, para que el círculo virtuoso avance, se requiere que las CVS se organicen legalmente, por ejemplo, en una sociedad de propósito especial o en un consorcio (punto 6).

Seguidamente, la actividad productiva presenta oportunidades para generar sostenibilidad vía la reducción del desperdicio (punto 7). Un añadido importante es la relativa facilidad de reflejar en los estados de resultados los avances de los procesos circulares, pues se trata de un modelo guiado por criterios de eficiencia con mejores márgenes económicos. Esa puede ser la base de la resiliencia necesaria para resolver los retos del cambio climático y de la pobreza estructural, a través de las finanzas (punto 8), y con ello, mejorar el bienestar del ecosistema (punto 9).

Como se observa, la estrategia que se plantea en esta investigación busca una perspectiva integradora, teniendo como foco el desarrollo de la CVS. Asimismo, tal como lo señalan Kell y Rasche (2020), las cadenas de valor también tienen como base a la relación entre economía y salud, así como entre economía y ecología.
El énfasis puesto en la relación humanidad y naturaleza se explica porque la estrategia para construir resiliencia a través de las CVS debe ser incorporada como un factor de riesgo no solo declarativo, sino como un riesgo a ser gestionado. Esto conduce a que los destinatarios de los recursos de fondos deban desarrollar habilidades para el tratamiento de este riesgo, lo que implica adquirir nuevas competencias y también tener más y mejores equipos multidisciplinarios. $\mathrm{Al}$ respecto, Berensmann y Lindenberg (2016) proponen la "ecologización" de los sistemas financieros, es decir, la actividad económica sostenible para financiar debe evidenciar su contribución al bienestar de su ecosistema de influencia para calificar al financiamiento.

A juicio de los autores, alcanzar lo señalado implica un reto por el lado de la oferta y demanda de recursos monetarios. Lo que hoy muestran los análisis realizados por los autores sobre gestión de negocios en Perú es que, por el lado de la oferta, no hay suficientes recursos, y por el lado de la demanda, la situación es aún más frágil. Se considera que institucionalizar las CVS en cualquier ámbito de la actividad económica sería una herramienta de mucha utilidad.

Un ejemplo para construir un modelo sostenible enfocado en una organización de base se presenta a través de los pequeños productores de jengibre orgánico del distrito de Pichanaqui (provincia de Chanchamayo, departamento 
de Junín, Perú) (Reátegui, 2021). El modelo busca llevar a los productores jengibre orgánico hacia mercados de mayor valor. El proyecto sigue los lineamientos de desarrollo sostenible e inversión de impacto, promoviendo el fortalecimiento de la organización vía la capitalización de modelos de negocios de agroexportación y la obtención de las utilidades generadas por toda la cadena de suministro.

En el modelo que se propone, no se ha identificado ninguna referencia a compartir excedentes o utilidades, pues nadie, aparte de la asociación, lidera el negocio, el riesgo, así como las ganancias; no existe una distribución del excedente, sino el pago de factores de producción o por servicios que apoyan al modelo de negocio; por ende, el manejo del efectivo del proyecto es $100 \%$ de la asociación.

De lo planteado en el caso del jengibre de Pichanaqui, surgen elementos y actores que deben ser considerados por las finanzas sostenibles y por las CVS, como son: 1) asociatividad $\mathrm{u}$ organizaciones de base; 2 ) modo de producción sostenible; 3 ) operación de un consorcio formal, legal, a lo largo de la cadena, de ida y vuelta con los derechos y obligaciones; 4) magnitud de la inversión necesaria para ir desde la producción primaria hasta un mercado de nuevos consumidores o consumidores de sostenibilidad que tiene claridad sobre sus beneficios y ventajas; 5) una adecuada gestión de la relación riesgo-retorno de cada eslabón de la cadena; y 6) gestión de los riesgos financieros y no financieros.

Sin embargo, se quiere hacer hincapié en el punto 5) adecuada gestión de la relación riesgo y retorno. Desde la perspectiva de esta investigación, por ejemplo, la producción primaria como la agricultura con sello orgánico implica mayores riesgos, empezando con los hidrometereológicos, la fragilidad institucional y la debilidad en la gestión comercial, la cual está expuesta a que otras partes se queden con el negocio generado y que, por lo general, no están comprometidos con la sostenibilidad.

Además del mayor riesgo que se señala, esta agricultura familiar es fuente del mayor valor de uso, pero con bajo valor de cambio, debido tal vez a la poca claridad al momento de establecer la relación riesgo y retorno. El primer eslabón de la cadena que se propone no compensa su riesgo con el retorno alcanzado en la fase inicial. Una respuesta eficiente para equilibrar la relación riesgo y retorno debe provenir de la operación de las CVS, y esa debe ser exigencia de las fuentes de financiamiento.

Como se señaló, se busca plantear modelos integradores de gestión que incorporen submodelos productivos, financieros y de comercialización como facilitadores de soluciones efectivas, con una visión compartida por los sectores público y privado y que incorpore a diferentes sectores del país, sobre la base de cadenas de valor en un formato de consorcio o de sociedad de propósito específico, cuya gestión integral contemple el bienestar expresado en las referencias a la salud planetaria y a los objetivos de desarrollo sostenible. Es decir, las CVS y el consorcio son los fundamentos para el planteamiento de un modelo de finanzas sostenibles.

Entonces, ¿por dónde comenzar? En primer lugar, se propone empezar por reconocer la necesidad de enlazar la producción potencial sostenible con nuevos hábitos de consumo responsable de mercados finales nacionales e internacionales, de manera ampliada, dejando el enfoque de nicho; es decir, se propone modelos de negocios agregados eficientes, con márgenes operacionales sólidos, gastos financieros bajos y costos de capital que reflejen riesgos acotados por una mejor gestión.

En segundo lugar, se debe reconocer la evidencia en relación con los agentes económicos que actúan como intermediarios de esa producción sostenible para colocarla en mercados finales, pues son los que se llevan la mayor parte de los excedentes generados (Reátegui, 2021). Esto limitaría severamente la capitalización de los productores, por lo tanto, la vigencia de una cadena de valor permite llegar al mercado desde las organizaciones de productores. Son varias las lecciones que deja y seguirá dejando la crisis sanitaria mundial, como migrar a un consumo responsable con inclusión financiera, y fortalecer a las personas más vulnerables, tal como lo proponen Quammen (2020) y Geissdoerfer et al. (2017).

Las relaciones que se observan en el modelo que se plantea pueden entenderse desde las finanzas corporativas, considerando las tres grandes decisiones empresariales: inversión, decisiones de financiamiento y de distribución de dividendos (Ross, Westerfield, Jaffe y Jordan, 2018). Con respecto a la tasa de descuento, lo "financieramente correcto" sería que esta tasa sea mayor, pues ir por el camino de la sostenibilidad implica un mayor riesgo financiero. Al respecto, Kling, Volz, Murinde y Ayas (2020) sostienen que la vulnerabilidad climática eleva el costo financiero debido a la restricción a las fuentes de financiamiento. De lo expresado, se presenta en la Figura 2 el modelo de gestión de negocios, considerando las finanzas sostenibles.

El módulo I describe los componentes que se requieren para enmarcar la decisión estratégica de invertir, estableciendo una primera línea de base que considera la estimación de las huellas de carbono, hídrica y ecológica. El módulo I establece las bases de un modelo de negocios sostenible que demandará inversiones, financiamiento, objetivos y metas sostenibles compartidas a lo largo de la CVS. 


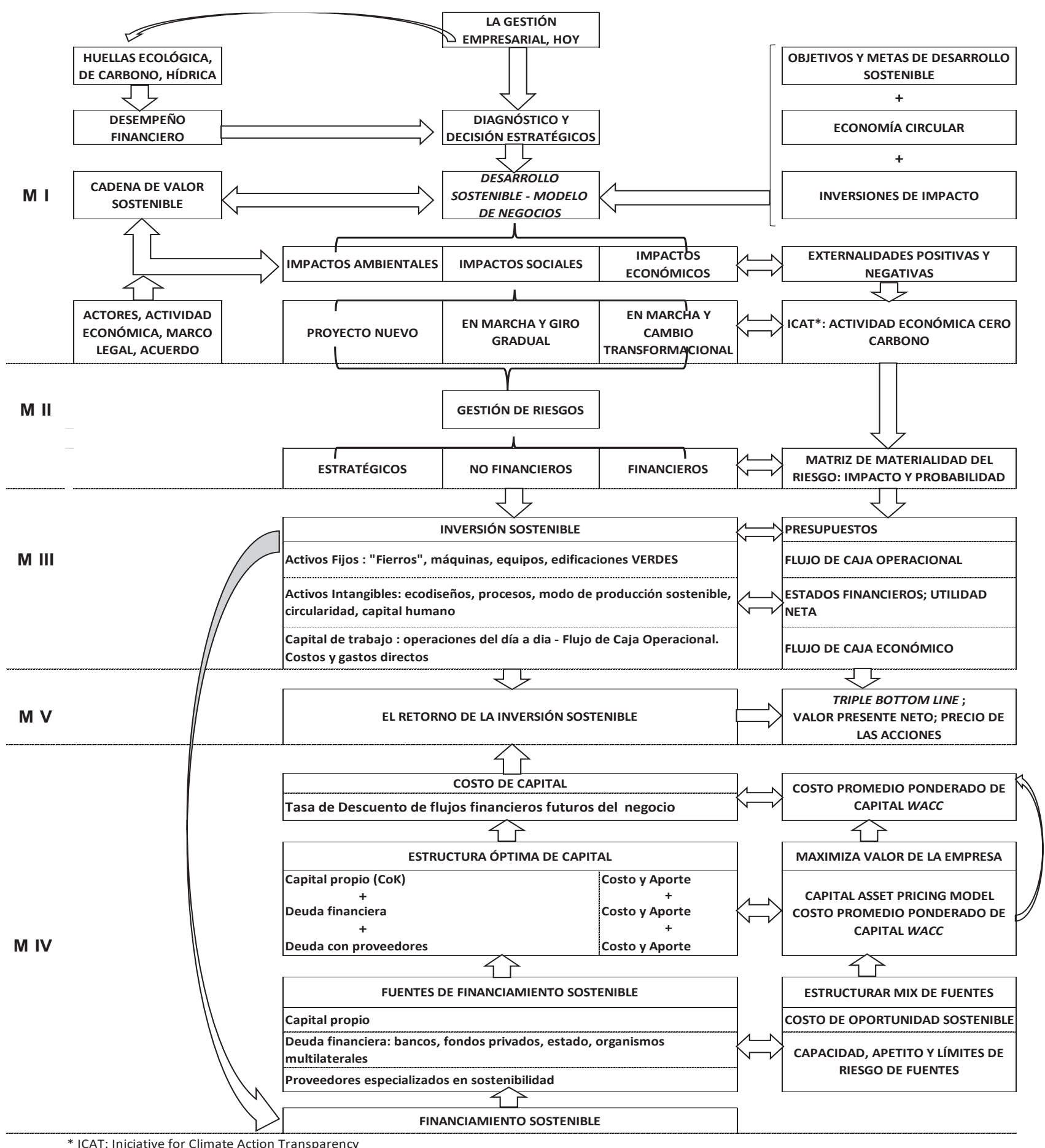

Figura 2. La gestión de la sostenibilidad y las finanzas

Fuente: Elaboración propia sobre la base de Reátegui (2021) 
El módulo II plantea la necesaria gestión de riesgos reflejada en la elaboración de una matriz generada por el proceso de identificar los eventos o factores de riesgo, estimar el impacto que podría tener en la gestión del negocio, así como la posibilidad de ocurrencia del evento. Esta identificación conduce a la gestión de todos los factores de riesgo estratégicos, operacionales y financieros, vía un conjunto de estrategias para mitigar impactos o transferir los riesgos en general.

La identificación de los riesgos requiere un sólido conocimiento del modelo operacional del negocio. Se puede apreciar tres rubros, siendo el primero la identificación del evento y complejidad, seguido de la valoración del impacto dado y de la estimación de su posibilidad de ocurrencia. Luego de este mapeo será posible plantear la gestión misma de cada factor a través de estrategias para mitigar el impacto. Es necesario poner énfasis en que las fuentes de financiamiento le dan un alto puntaje a solicitudes de crédito que muestran la materialidad de los riesgos del negocio. Un ejemplo de identificación de factores de riesgo se muestra en la Tabla 1.

El módulo III del modelo de gestión describe los componentes de la inversión sostenible. En esta sección, se debe destacar más allá de la naturaleza de los componentes de la inversión, activos fijos, activos intangibles y capital de trabajo, a los proveedores de bienes y servicios que incorporen la sostenibilidad, incluyendo financiamiento customizado. Es en este módulo en el que deben ser formulados presupuestos, flujos de caja operacional, estados financieros, el flujo de caja económico; así como esbozar las primeras líneas del costo de capital de la inversión, o costo de oportunidad del inversionista.

El módulo IV esquematiza el financiamiento de la inversión sostenible, haciendo referencia a los conceptos de fuentes y costos que deben conducir a la estructura óptima de capital, al flujo de caja financiero, al costo promedio ponderado de capital, valor actual neto y la tasa interna de retorno. El rol de las fuentes de financiamiento requiere ser destacado, pues sus capacidades de gestión o tolerancias al riesgo serán determinantes para avanzar hacia la sostenibilidad.

Finalmente, en el módulo $\mathrm{V}$, se presentan los resultados finales de la inversión sostenible vía indicadores de sostenibilidad y financieros, reducción de las huellas hídrica, ecológica y ambiental; la reducción de impactos y de externalidades. Juntamente con un mejor desempeño financiero, le dan sentido y valor al modelo de finanzas sostenibles. Así, ganan a través de un solo esfuerzo la sociedad y el planeta.

\section{DISCUSIÓN}

La crisis sanitaria provocada por el COVID-19 ha puesto de manifiesto la relación fehaciente entre medioambiente, salud y economía. Sin embargo, este contexto de crisis brinda también la posibilidad de cambios sustanciales en la gestión empresarial, introduciendo una visión de sostenibilidad, ya que, de no hacerlo, la aparición de nuevos virus es altamente probable, tal como lo señalan Quammen (2020), Prüss-Üstün et al. (2006), así como, Kell y Rasche (2020).

Si bien se han desarrollado métodos para apoyar la realización de negocios inclusivos, pareciera que elementos, como el cambio climático, no han sido incorporados adecuadamente, según Schoenmaker y Schramade (2019), Ziolo et al. (2019), y Bianchini y Gianfrate (2018). A esta posición se suma la de Bonini y Swartz (2014), quienes sostienen que lograr la sostenibilidad de los negocios, no debe ser visto como un innecesario aumento de costos, sino como una posibilidad para lograr réditos para todos.

Estos argumentos coinciden con el resultado de la investigación que considera que los efectos del cambio climático

Tabla 1

Identificación, valoración de impactos y gestión de factores de riesgo

\begin{tabular}{|c|c|c|c|c|c|}
\hline \multicolumn{2}{|c|}{ Identificación de factores de riesgo } & \multicolumn{2}{|c|}{ Valoración del factor (matriz de materialidad) } & \multicolumn{2}{|c|}{ Gestión (estrategia) del factor de riesgo } \\
\hline $\begin{array}{l}\text { Factor de riesgo / } \\
\text { subfactores }\end{array}$ & Grado de criticidad & Impacto esperado & $\begin{array}{l}\text { Posibilidad de } \\
\text { ocurrencia }\end{array}$ & $\begin{array}{l}\text { Mitigación / control } \\
\text { de daños }\end{array}$ & Transferencia \\
\hline $\begin{array}{l}\text { Actividad que con- } \\
\text { lleva de manera } \\
\text { expresa la posibili- } \\
\text { dad de falla. }\end{array}$ & $\begin{array}{l}\text { Nivel del impacto } \\
\text { esperado: a mayor } \\
\text { impacto, mayor } \\
\text { criticidad. Podría } \\
\text { detener un nego- } \\
\text { cio o proyecto. }\end{array}$ & $\begin{array}{l}\text { Valor en unidades } \\
\text { monetarias del im- } \\
\text { pacto: gasto, menor } \\
\text { ingreso, pérdida. } \\
\text { Afecta resultado final. }\end{array}$ & $\begin{array}{c}\text { Asignar un grado de } \\
\text { ocurrencia del factor } \\
\text { o evento, desde bajo } \\
\text { hasta alto. }\end{array}$ & $\begin{array}{l}\text { Acción predefinida } \\
\text { de control para } \\
\text { reducir impacto } \\
\text { negativo: no sale de } \\
\text { la empresa. }\end{array}$ & $\begin{array}{c}\text { Acción predefinida } \\
\text { para eliminar im- } \\
\text { pacto negativo a un } \\
\text { costo conocido: sale } \\
\text { de la empresa. }\end{array}$ \\
\hline
\end{tabular}

Fuente: Elaboración propia 
no se habrían abordado desde una perspectiva financiera moderna, ni se habrían reconocido los riesgos que generan en la cadena de valor de un negocio, lo que impide hacer una adecuada evaluación de riesgo y retorno, dificultando el acceso al financiamiento, provocando que el mayor excedente no quede en manos de las poblaciones más frágiles, y manteniéndose estas en un círculo de pobreza del que no pueden salir. Al respecto, Berensmann y Lindenberg (2016) proponen la "ecologización" de los sistemas financieros, frase que captura la necesidad de cambios urgentes.

Por otro lado, las poblaciones vulnerables no solo deben ser vistas como parte de la demanda en el mercado, sino como una fuente generadora de riqueza, tal como los señalan Nidumolu et al. (2009) y Reátegui (2021). En este contexto, se propone la configuración de las CVS, donde los actores y sus funciones estén claramente identificados, y donde las finanzas sean abordadas bajo nuevos paradigmas.

Las finanzas sostenibles deben alinearse en mayor medida hacia los objetivos de apoyo a las personas con mayor fragilidad. Las inversiones serán sostenibles si se puede lograr que estas comunidades puedan desarrollar capacidades para los negocios, incorporando habilidades para identificar adecuadamente los riesgos. Asimismo, según Reátegui (2021), es clave que las fuentes de financiamiento profundicen en modelos de evaluación crediticia donde, por ejemplo, la gestión de los riesgos ambientales sea claramente incorporada. Los resultados de una gestión innovadora deben verse reflejados en los estados financieros de los emprendimientos que se realicen.

Como respuesta a la pregunta de investigación, se concluye que, si bien las finanzas son consideradas en los emprendimientos de las personas menos favorecidas, no se ajustan a un buen balance de riesgo y rentabilidad de los actores en la CVS, contribuyendo a que los más vulnerables no puedan emerger de situaciones de pobreza. Por lo tanto, como producto de esta investigación, se estableció claramente la relevancia de las finanzas dentro de un modelo de negocios sostenibles para poblaciones vulnerables. En el modelo conceptual diseñado, se propone una gestión integrada que equilibre la relación ortodoxa entre riesgo y retorno, trasladando más retorno a quien más riesgo gestiona. En el modelo, no debe existir una distribución del excedente, sino el pago de factores de producción o por servicios que apoyen al modelo de negocio. Esta gestión debe estar $100 \%$ bajo responsabilidad de la comunidad vulnerable, con apoyo de gestores que les soporten en la identificación adecuada de los riesgos.

Conciliar el bienestar de productores primarios asociados, mitigación y adaptación al riesgo de cambio climático, finanzas sostenibles, inclusión financiera, acceso a mercados de consumidores alineados con objetivos de bienestar general, entre otros, representa un reto que demanda nuevas capacidades, compromisos y recursos monetarios, por lo que se considera indispensable la cooperación de las políticas públicas, la participación de los fondos multilaterales, fondos privados y las universidades.

Luego de plasmar el modelo de negocios sostenible, se recomienda concluir su implementación dentro de la comunidad de Pichanaqui. Los resultados a los que se llegue permitirán identificar mejoras al modelo conceptual presentado para que pueda ser discutido con otras organizaciones de base, las fuentes de financiamiento y la academia.

Para futuras investigaciones, se propone absolver las si-

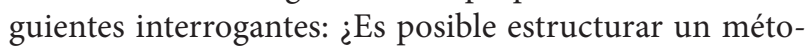
do que permita la determinación de la tasa de descuento que logre un adecuado balance entre riesgo y rentabilidad, incorporando los riesgos del cambio climáticos que afectan a las poblaciones más vulnerables? Asimismo, ¿es posible la implementación de las cadenas de valor sostenible en la minería artesanal, entendiendo que esta actividad se realiza intensamente en zonas de conservación de la biodiversidad?

\section{REFERENCIAS}

Acero, L., \& Savaget, P. (29 de octubre de 2014). Plural Understandings of Sociotechnical Progress within the OECD [conferencia]. 12 $2^{\text {th }}$ Globelics International Conference, Adís Abeba, Etiopía.

Barrientos, P. (2018). La agricultura peruana y su capacidad de competir en el mercado internacional. Equidad y Desarrollo, 1(32), 143-179. https://doi.org/10.19052/ $\underline{\text { ed. } 5056}$

Berensmann, K., \& Lindenberg, N. (2016). Green Finance: Actors, Challenges, and Policy Recommendations. Briefing Paper, (23), 1-4 Recuperado de http://hdl. handle.net/10419/199787

Bianchini, R., \& Gianfrate, G. (2018). Climate Risks and the Practice of Corporate Valuation. En S. Boubaker, D. Cumming, \& D. K. Nguyen (Eds.), Research Handbook of Finance and Sustainability (pp. 436-457). https://doi.org/10.4337/9781786432636.00032

Bonini, S., \& Swartz, S. (2014). Profits with Purpose: How Organizing for Sustainability Can Benefit the Bottom Line. McKinsey on Sustainability \& Resource Productivity, 2(1), 5-15. Recuperado de https://www.mckinsey.com/ /media/McKinsey/Business\%20Functions/ Sustainability/Our\%20Insights/Profits\%20with\%20 purpose/Profits\%20with\%20Purpose.ashx 
Ellen MacArthur Foundation. (2013). Towards the Circular Economy: Opportunities for the Consumer Goods Sector [vol. 2]. Recuperado de https://ellenmacarthurfoundation.org/towards-the-circular-economy-vol-2opportunities-for-the-consumer-goods

Geissdoerfer, M., Savaget, P., Bocken, N. M., \& Hultink, E. J. (2017). The Circular Economy - A New Sustainability Paradigm? Journal of Cleaner Production, (143), 757-768. https://doi.org/10.1016/j.jclepro.2016.12.048

Kell, G., \& Rasche, A. (2020). Renewing Markets from Within: How Businesses and the Investment Community Can Drive Transformational Change. En H. Bril, G. Kell, \& A. Rasche (Eds.), Sustainable Investing: A Path to a New Horizon (pp. 45-66).

Kling, G., Volz, U., Murinde, V., \& Ayas, S. (2020). The Impact of Climate Vulnerability on Firmas' Cost of Capital and Access to Finance. World Development, (137), 1-11. https://doi.org/10.1016/j.worlddev.2020.105131

Nidumolu, R., Prahalad, C. K., \& Rangaswami, M. R. (2009). Why Sustainability is Now the Key Driver of Innovation. Harvard Business Review, 87(9), 56-64. Recuperado de https://hbr.org/2009/09/why-sustainability-is-now-the-key-driver-of-innovation

Prüss-Üstün, A., Corvalán, C. F., \& World Health Organization. (2006). Ambientes saludables y prevención de enfermedades. Hacia una estimación de la carga de morbilidad atribuible al medioambiente. Resumen de orientación. Recuperado de https://apps.who.int/iris/ handle/10665/43452
Quammen, D. (2020). Contagio. La evolución de las pandemias. Barcelona: Debate.

Reátegui, W. (2021). Finanzas sostenibles. De la vulnerabilidad hacia la resiliencia. Recuperado de https://bit. ly/3Cg4wk1

Ross, S., Westerfield, R., Jaffe, J., \& Jordan, B. (2018). Finanzas corporativas (11. ${ }^{\mathrm{a}}$ ed.). México D.F.: McGraw Hill.

Schoenmaker, D., \& Schramade, W. (2018). Principles of Sustainable Finance. Oxford: Oxford University Press.

Semieniuk, G., Campiglio, E., Mercure, J. F., Volz, U., \& Edwards, N. R. (2021). Low-Carbon Transition Risks for Finance. Wiley Interdisciplinary Reviews: Climate Change, 12(1), 1-24. https://doi.org/10.1002/wcc.678

Stahel, W. (2019). The Circular Economy: A User's Guide. Londres: Routledge.

Ziolo, M., Filipiak, B. Z., Bąk, I., Cheba, K., Tîrca, D. M., \& Novo-Corti, I. (2019). Finance, Sustainability and Negative Externalities. An Overview of the European Context. Sustainability, 11(15), 1-35. https://doi. org/10.3390/su11154249 